\title{
Capnography Detects Phrenic Nerve Stimulation during Cryothermal Ablation
} O’Neill DK ${ }^{*}$, Aizer A ${ }^{2}$, Linton $\mathrm{PL}^{3}$, Chinitz $\mathrm{L}^{4}$ and Blanck $\mathrm{T}^{5}$

${ }^{1}$ Assistant Professor of Anesthesiology, Associate Director of Adult Off-Site Anesthesiology Services, New York University School of Medicine, New York University Langone Medical Center, New York, NY, USA ${ }^{2}$ Assistant Professor of Medicine, Associate Director of Heart Rhythm Center, New York University School of Medicine, New York University Langone Medical Center, New York, NY, USA

${ }^{3}$ Assistant Professor of Anesthesiology, Director of Adult Off-Site Anesthesiology Services and Endoscopy, New York University School of Medicine, New York University Langone Medical Center, New York, NY, USA ${ }^{4}$ Professor of Medicine, Director of Heart Rhythm Center, New York University School of Medicine, New York University Langone Medical Center, New York, NY, USA

${ }^{5}$ Professor of Anesthesiology, Professor, Department of Neuroscience Institute, New York University School of Medicine, New York University Langone Medical Center, New York, NY, USA

${ }^{*}$ Corresponding author: O'Neill DK, Associate Director, Adult Offsite Anesthesiology Services, Assistant Professor of Anesthesiology, Department of Anesthesiology, Perioperative Care and Pain Medicine, New York

Citation: O’Neill DK, Aizer A, Linton PL, Chinitz L, Blanck T (2017) Capnography Detects Phrenic Nerve Stimulation during Cryothermal Ablation. J Anesth Pati Care 2(1):101. doi: 10.15744/2456-5490.2.101

Received Date: February 04, 2017 Accepted Date: February 28, 2017 Published Date: March 01, 2017

\section{Images in Anesthesiology}

\section{Case Presentation}

A 54 year old female presented for atrial fibrillation (AF) catheter cryoablation. She had experienced multiple pre-syncopal episodes prior to diagnosis and underwent a cardiac work-up including Holter monitor, transthoracic echocardiogram,stress test and tilt table test which was negative except for atrial fibrillation. She presented to the hospital for an AF ablation procedure under general anesthesia after transesophageal echocardiogram to rule out atrial thrombosis formation.

The patient was brought to the cardiac electrophysiology laboratory with a 20 gauge intravenous line in place in the left antecubital fossa. Standard ASA monitors and a BIS monitor were placed prior to induction. After induction of anesthesia, with propofol 250 $\mathrm{mcg} / \mathrm{kg} / \mathrm{min}$ x $5 \mathrm{~min}$, remifentanil $0.05 \mathrm{mcg} / \mathrm{kg} / \mathrm{min}$ x $5 \mathrm{~min}$, fentanyl $250 \mathrm{mcg}$, rocuronium $50 \mathrm{mg}$, intubation was performed and placement was confirmed with end-tidal carbon dioxide $\left(\mathrm{ETCO}_{2}\right)$. An esophageal temperature probe and a gauze bite block were also placed. Anesthesia was maintained with remifentanil and propofol infusions for duration of the procedure. The depth of sedation with propofol was titrated to maintain a BIS of 45-55 throughout. Neuromuscular blockade was not maintained after induction to allow phrenic nerve monitoring during phrenic nerve pacing with a 3.5-mm-tip catheter (ThermoCool NaviStar, Biosense Webster) to determine ablation catheter misplacement.

At the time of cryoablation and phrenic nerve monitoring, the capnogram demonstrated interruption of normal exhalation (Figure 1) consistent with diaphragmatic movement and preservation of phrenic nerve integrity. The ETCO2 capnogram demonstrated the classic "curare cleft" and the flow sensor triggered a pressure support breaths demonstrated by vertical line in flow and pressure waves. There was a decrease in $\mathrm{EtCO}_{2}$ and change in slope of the capnogram. The cooling from the cryoablation was also noted with a 7-10 degree Centigrade drop in measured temperature at the tip of the esophageal probe.

The case proceeded uneventfully and the patient was extubated and taken to PACU without incident.

\section{Discussion}

Atrial fibrillation is the most common arrhythmia affecting more than 5 million Americans. 1 Treatment options have focused on controlling the patient's heart rate or rhythm. However, many pharmacological therapies are ineffective or intolerable due to side effects. Ablation therapy, which is targeted myocyte necrosis, has been widely used to treat this condition. Ablation is most frequently performed via heating the tissue with radiofrequency energy. Alternatively, ablation can be performed by freezing tissue, typically called cryoablation. Ablation therapy for atrial fibrillation is typically aimed at the myocytes located within pulmonary veins where AF substrate and foci most commonly arise. 

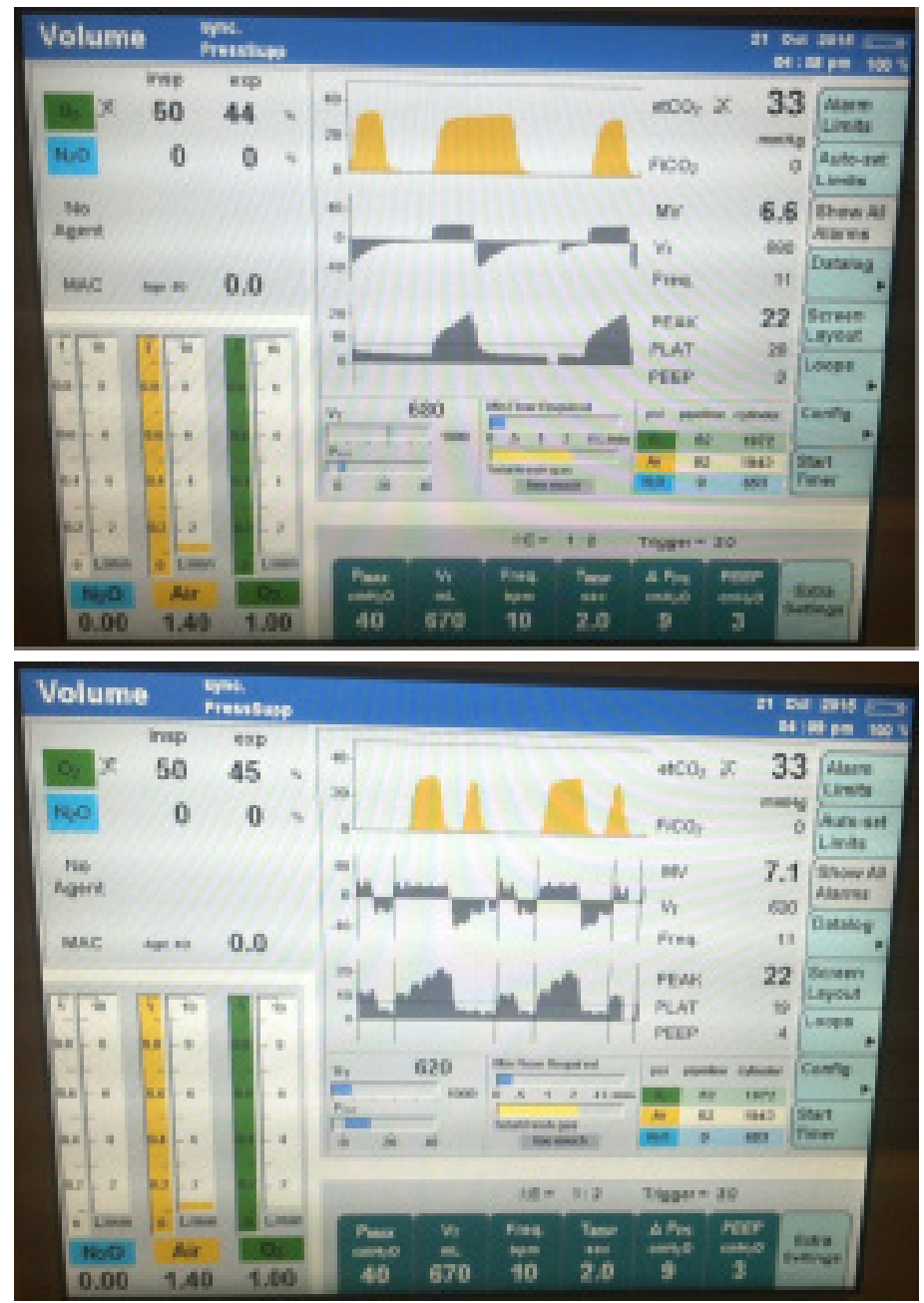

The $\mathrm{ETCO}_{2}$ capnogram demonstrated the classic "curare cleft" and the flow sensor triggered a pressure support breaths demonstrated by vertical line in flow and pressure waves

Figure 1: Capnogram, Flow, and Pressure Waves Before and After Phrenic Nerve Pacing

The right phrenic nerve runs between the SVC posteriorly and the right upper pulmonary vein; it then proceeds anterolaterally to the RA/SVC junction (Figure 2). Phrenic nerve pacing with observation of diaphragmatic movement is frequently performed to assure that the heated or cooled catheter is not injuring the phrenic nerve during ablations of the SVC or right upper pulmonary vein (RUPV), atrial tachycardia and epicardial ventricular tachycardia ablations, or biventricular ICD placements. The phrenic nerve can be damaged while ablation therapy is performed due to the proximity of the phrenic nerve to the AF ablation energy source. 3 When ablation is performed, the right phrenic nerve is frequently stimulated with high output pacing from the SVC in order to monitor function of this nerve. Ablation is terminated if phrenic nerve stimulation of diaphragmatic movement becomes attenuated. Cryoablation is different from RF ablation in that, the loss of electrical activity is reversible at - 30 degrees centigrade. When right sided cryoablation is performed, the catheter is cooled and diaphragmatic stimulation is performed via the ipsilateral phrenic nerve. If diaphragmatic movement is attenuated despite phrenic nerve stimulation proximal to the ablation location, cooling is discontinued and phrenic nerve function typically returns (Figure 3 ).

Anesthetic considerations include the monitoring the integrity of neuromuscular junction function while phrenic nerve stimulation is being performed. In each of these scenarios, continued neuromuscular blockade would obviate the usefulness of phrenic nerve monitoring and increase the risk of phrenic nerve injury from the procedure. These patients are usually under general anesthesia or deep sedation in order to tolerate mapping and ablation of AF. It is critical that patients have intact nerve function and therefore, do not have muscle relaxant effects during phrenic nerve stimulation. As anesthesiologists who often work outside of the operating room, we need to be familiar with these emerging therapies. Due to our physical separation from the operating room, there is a great chance that we may not have trained personnel in our immediate vicinity that can differentiate true anesthetic emergencies from anticipated complications.

It is important to recognize that diaphragmatic movement will be detected in the capnograph and this does not signify light anesthesia and/or voluntary patient movement. Therefore, the phrenic nerve stimulation of the diaphragm should not be treated with neuromuscular blockade in these cases. While phrenic nerve injury typically resolves, the injury frequently persists for up to one year and may not always resolve. The elevated hemidiaphragm frequently leads to dyspnea on exertion. 

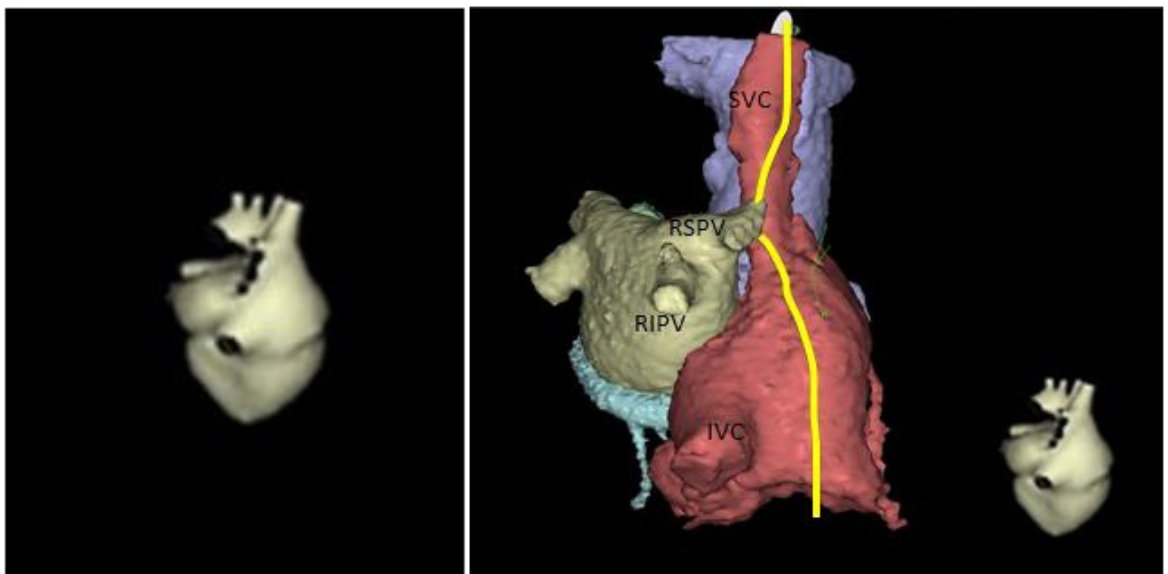

A right posterior oblique view of the right atrium (red), left atrium (gray), aorta (blue) and coronary sinus (green). The right phrenic nerve (yellow) descends along the right anterior-lateral border of the superior vena cava (SVS). Along its course, the nerve curves posteriorly around the SVC towards the right pulmonary veins, closest to the right superior pulmonary vein (RSPV), but also within proximity of the right inferior pulmonary vein (RIPV). The nerve then proceed along the anterior-lateral right atrium Figure 2: Typical Course of Right Phrenic Nerve

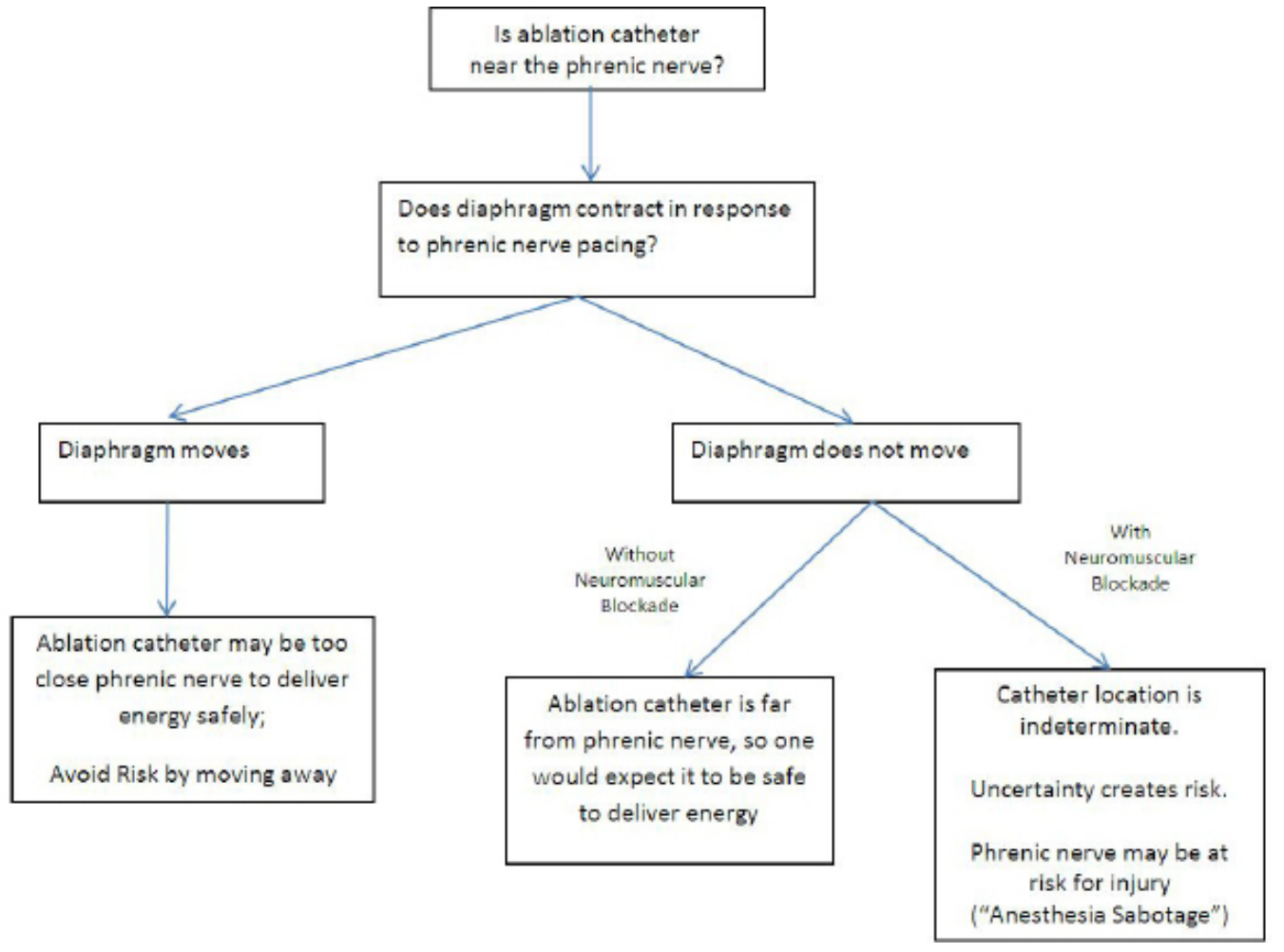

Figure 3: Algorithm for Phrenic Nerve Pacing

\section{References}

1. Go AS, Hylek EM, Phillips KA, Chang YC, Henault LE, et al. (2001) Prevalence of diagnosed atrial fibrillation in adults: national implications for rhythm management and stroke prevention: the Anticoagulation and Risk Factors in Atrial Fibrillation (ATRIA) Study. JAMA 285: 2370-5.

2. Sarabanda AV, Bunch TJ, Johnson SB, Mahapatra S, Milton MA, et al. (2005) Efficacy and safety of circumferential pulmonary vein isolation using a novel cryothermal balloon ablation system. J Am Coll Cardiol 46: 1902-12.

3. Dib C, Kapa S, Powell BD, Packer DL, Asirvatham SJ (2008) Successful use of "cryo-mapping" to avoid phrenic nerve damage during ostial superior vena caval ablation nerve proximity. J Interv Card Electrophysiol 22: 23-30.

4. Sarabanda AV, Bunch TJ, Johnson SB, Mahapatra S, Milton MA, et al. (2005) Efficacy and safety of circumferential pulmonary vein isolation using a novel cryothermal balloon ablation system. J Am Coll Cardiol 46: 1902-12.

5. Medtronic (2013) Arctic Front Cardiac CryoAblation Catheter clinical reports, in support of FDA premarket approval, Medtronic Inc., USA.

6. Kojodjojo P1, O'Neill MD, Lim PB, Malcolm-Lawes L, Whinnett ZI, et al. (2010) Pulmonary venous isolation by antral ablation with a large cryoballoon for treatment of paroxysmal and persistent atrial fibrillation: medium-term outcomes and non-randomised comparison with pulmonary venous isolation by radiofrequency ablation. Heart 96: 1379-84.

7. Van Belle Y, Janse P, Theuns D, Szili-Torok T, Jordaens L (2008) One year follow-up after cryoballoon isolation of the pulmonary veins in patients with paroxysmal atrial fibrillation. Europace 10: 1271-6. 
8. Schmidt B, Chun KRJ, Ouyang F, Metzner A, Antz M, et al. (2008) Three-dimensional reconstruction of anatomic course of the right phrenic nerve in humans by pace mapping. Heart Rhythm 5: 1120-6.

9. Andrade JG, Khairy P, Dubuc M (2013) Catheter Cryoablation Biology and Clinical Uses. Circ Arrhythm Electrophysiol 6: 218-27.

10. Lee BK, Choi KJ, Kim J, Rhee KS, Nam GB, et al. (2004) Right phrenic nerve injury following electrical disconnection of the right superior pulmonary vein. Pacing Clin Electrophysiol 27: 1444-6.

11. Sacher F, Monahan KH, Thomas SP, Davidson N, Adragao P, et al. (2006) Phrenic nerve injury after atrial fibrillation catheter ablation: characterization and outcome in a multicenter study. J Am Coll Cardiol 47: 2498-503.

12. Matsumoto Y, Krishnan S, Fowler SJ, Saremi F, Kondo T, et al. (2007) Detection of Phrenic Nerves and Their Relation to Cardiac Anatomy Using 64-Slice Multidetector Computed Tomography. Am J Cardiol 100: 133-7.

13. Sanchez-Quintana D, Cabrera JA, Climent V, Farre J, Weiglein A, et al. (2005) How close are the phrenic nerves to cardiac structures? Implications for cardiac interventionalists. J Cardiovasc Electrophysiol 16: 309-13.

14. Bunch TJ, Bruce GK, Mahapatra S, Johnson SB, Miller DV, et al. (2005) Mechanisms of phrenic nerve injury during radiofrequency ablation at the pulmonary vein orifice. J Cardiovasc Electrophysiol 16: 1-8.

15. Randhawa A, Sahni D, Aggarwal A, Rohit MK, Sehgal S, et al. (2014) Study of Spatial Relationship of Phrenic Nerves with Cardiac Structure Relevant to Electrophysioloic Interventions. PACE 37: 1477-84.

16. Horton R, DiBiase L, Reddy V, Neuzil P, Mohanty P, et al. (2010) Locating the right phrenic nerve by imaging the right pericardiophrenic artery with computerized tomographic angiography: Implication for balloon-based procedures. Heart Rhythm 7: 937-41.

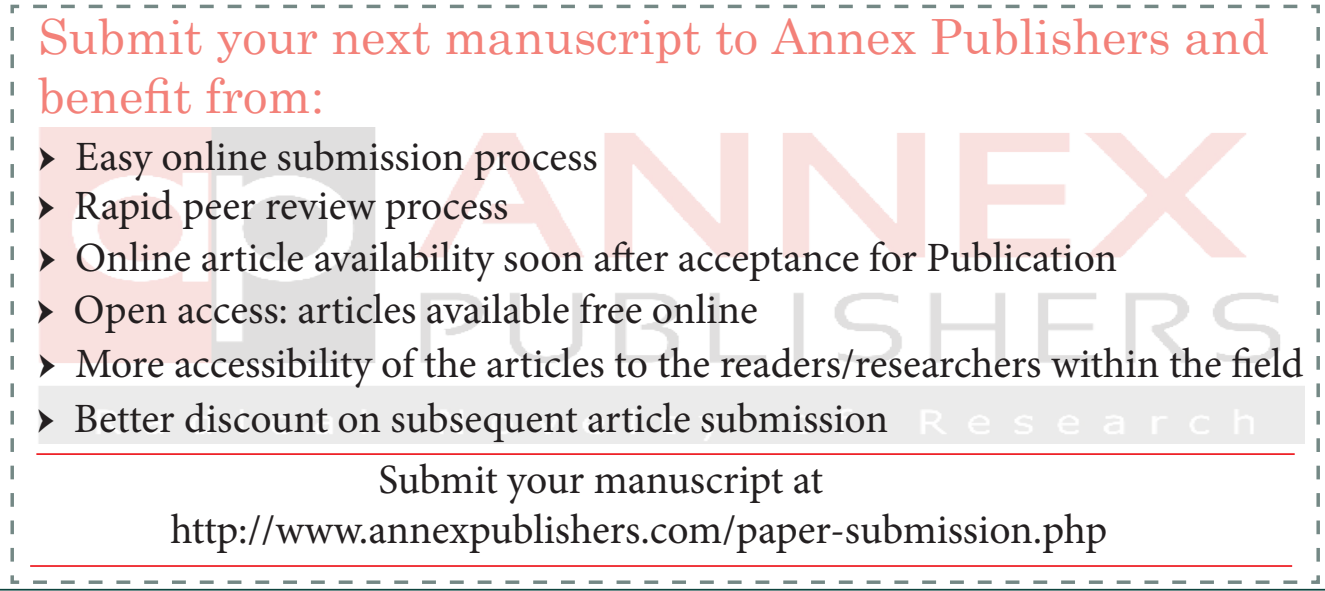

